\title{
Continuous True-Time-Delay Beamforming for Phased Array Antenna Using a Tunable Chirped Fiber Grating Delay Line
}

\author{
Yunqi Liu, Jianliang Yang, and Jianping Yao, Senior Member, IEEE
}

\begin{abstract}
In this letter, we demonstrate a new five-element true-time-delay (TTD) system that uses only a single tunable linear chirped fiber grating (TLCFG) delay line for continuous TTD beamforming. The system avoids the complicated wavelength tuning and synchronal control of the tunable lasers as well as the tunable bandpass filters. Different time delays are achieved by tuning the chirp rate of the TLCFG, to which a multiwavelength laser source that carries the RF signal is applied. The time delay responses of the TTD system are measured experimentally, which agree well with the theoretical analysis. The beampointing angle can be controlled continuously by changing the chirp rate of the TLCFG. The minimum time delay step can be much smaller than $1 \mathrm{ps}$ thanks to the high position resolution of the actuator.
\end{abstract}

Index Terms-Chirped fiber grating, chirp rate, continuously tunable, fiber delay line, true-time-delay.

\section{INTRODUCTION}

D URING last few years, photonic true-time-delay (TTD) units have been widely investigated for wideband squint-free beamforming for phased array antennas (PAAs). The advantages provided by TTD beamforming technique include low loss, small size, lightweight and immunity to electromagnetic interference. Among different TTD configurations, fiber grating prism (FGP) is considered a promising technique to achieve wideband photonic TTD beamforming [1]-[7]. Two approaches are usually employed to construct an FGP beamsteerer, the first one uses discrete fiber Bragg grating (FBG) delay lines to achieve discrete beamsteering at microwave frequencies less than $3 \mathrm{GHz}$ [1], [3]. The second one employs a chirped fiber grating to achieve continuous beamsteering at higher microwave frequencies [5], [6]. TTD units based on the chirped fiber grating delay line using tunable multiwavelength lasers and tunable bandpass filters have been reported and demonstrated [5], [6]. In the approach, different time delays are obtained by tuning the wavelengths of the optical carriers that are reflected by the chirped grating at different locations. The use of just one fiber chirped grating for the whole system avoids the time delay inaccuracies induced by the

Manuscript received January 25, 2002; revised March 29, 2002.

Y. Liu was with the Photonics Research Group, School of Electrical and Electronic Engineering, Nanyang Technological University, Singapore 639798 (e-mail: eyqliu@ntu.edu.sg). He is now with the School of Engineering, City University, ECIV OHB London, U.K. (e-mail: y.liu@city.ac.uk).

J. Yang is with the Photonics Research Group, School of Electrical and Electronic Engineering, Nanyang Technological University, Singapore 639798.

J. Yao was with the Photonics Research Group, School of Electrical and Electronic Engineering, Nanyang Technological University, Singapore 639798. He is now with the School of Information Technology and Engineering, University of Ottawa, KIN 6N5 Ottawa, Canada.

Publisher Item Identifier S 1041-1135(02)06270-5. position mismatches of many gratings. But the system is very complicated since one needs to use multiwavelength tunable laser sources with wavelengths controlled by a programmable wavelength controller. In addition, the tunable bandpass filters should also be tuned synchronously with wavelengths of the tunable laser source. To eliminate these disadvantages, a variable time delay line has been used for PAA system, which applies strain perturbation at different positions of a uniform FBG to get different time delays [8]. The system operates at a single fixed optical wavelength, but complicated automatic and electrical modules are required to control the perturbation in the fiber grating. The minimum time delay step is limited to about 10 ps by the minimum available distance between the adjacent perturbations.

In this letter, we propose and demonstrate a new true-timedelay system that uses only a single tunable linear chirped fiber grating (TLCFB) delay line to achieve continuous TTD beamforming. The system avoids the complicated wavelength tuning and the synchronal control of the tunable lasers as well as the tunable bandpass filters. Different time delays are achieved by tuning the chirp rate of the TLCFG, to which a multiwavelength laser source that carries the RF signals is applied. A five-element TTD unit is constructed and experimented. The time delay responses of the TTD system are measured experimentally, which agree well with the theoretical analysis. The beampointing angle can be controlled continuously by changing the chirp rate of the chirped grating. In the experiments, the chirp rate of the TLCFG is tuned by changing the spectral width of a uniform FBG surface-mounted on a simply supported beam. The minimum time delay step can be much smaller than $1 \mathrm{ps}$, which is determined by the position resolution of the high precision actuator and the wavelength spacing of the optical carriers.

\section{PRINCIPLE AND THEORY}

The system configuration of the five-element TTD unit employing TLCFG delay line is illustrated in Fig. 1. The light of the multiwavelength laser is externally modulated by a microwave signal through an electrooptic modulator (EOM). The output of the laser source has five fixed wavelengths with identical wavelength spacing. A polarization controller (PC) is inserted between the laser and the EOM to control the polarization state. The modulated light is sent to the TLCFG through an optical circulator, and then reflected by the TLCFG. The reflected light feeds a group of $N(N=5)$ single-mode fibers through an equal-path 1: $N$ power divider. Each channel includes a bandpass filter whose center wavelength coincides with one of the $N$ wavelengths of the laser source. For our system, the wavelengths of the laser are $\lambda_{0}-2 \Delta \lambda, \lambda_{0}-\Delta \lambda, \lambda_{0}, \lambda_{0}+\Delta \lambda, \lambda_{0}+2 \Delta \lambda$, 


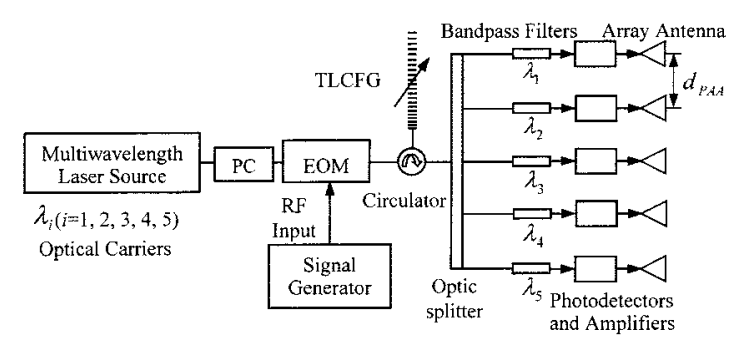

Fig. 1. Configuration of a five-element TTD system using TLCFG delay line.

where $\lambda_{0}$ is the center wavelength of the TLCFG and $\Delta \lambda$ is the wavelength spacing between adjacent wavelengths of the multiwavelength laser source. In the experiments, the chirp rate of the TLCFG is tuned continuously and the optical signals are reflected back by the TLCFG at different locations. The different time delays are obtained in accordance with the particular carrier wavelengths for $N$ different channels. The $N$ delayed modulated light is then detected by $N$ high-speed photodetectors and are sent after amplification to feed $N$ antenna-radiator elements. To ensure an acceptable signal-to-noise ratio (SNR) at the outputs of the photodetectors, an erbium-doped fiber amplifier (EDFA) is incorporated into the system after the modulator to compensate for the insertion loss and $N$ microwave preamplifiers are also adopted after the photodetectors to amplify the detected RF signal.

For the TTD system shown in Fig. 1, the beampointing angle corresponding to the main lobe of the array antenna, $\theta_{0}$, can be expressed as

$$
\sin \theta_{0}=\frac{c \cdot \Delta t_{d}}{d_{\mathrm{PAA}}}
$$

where $d_{\mathrm{PAA}}$ is the element spacing of antenna array, $c$ is the free-space speed of light, and $\Delta t_{d}$ is the time delay difference between adjacent channels. The equation states that the beampointing direction is determined by the time delay difference and is independent of the microwave frequency. Therefore, the TLCFG delay line is a true-time-delay beamformer and is suitable for wideband applications. For our system, the time delay difference is determined by the chirp rate of the chirped grating and the wavelength spacing of the optical carriers. It can be expressed as

$$
\Delta t_{d}=k_{\text {chirp }} \cdot \Delta \lambda
$$

where $k_{\text {chirp }}$ is the chirp rate of the TLCFG. Being different from other chirped-grating-based TTD units, in the proposed TTD system shown in Fig. 1, the wavelength spacing of optical carrier is fixed while the chirp rate of the grating is tuned continuously. The time delay difference is linear with the chirp rate and the beampointing direction is determined by the chirp rate of the TLCFG.

Several techniques have been proposed to produce adjustable chirped fiber gratings. Although the other techniques can also be used to demonstrate the operation of the above TTD system, in the proposed approach, we employ the simply supported beam tuning technique to produce the adjustable linear chirped grating [9]. This technique allows the continuously dynamic control of the spectral width of a uniform FBG while the center wavelength is kept unshifted. Fig. 2 shows the schematic configuration of the chirp tuning setup. The uniform FBG is surface-mounted on one side of a simply supported beam. The midpoint of the

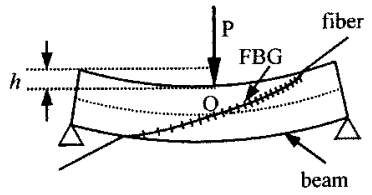

Fig. 2. Schematic configuration of the FBG chirp tuning setup.

grating is consistent with the center of the cross section $O$. The beam is bent under load $P$ at point $O$. The spectral width of the chirped grating, $\Delta \lambda_{G}$, can be expressed as [9]

$$
\Delta \lambda_{G}=k h
$$

where $k$ is a constant determined by the parameters of the beam and the FBG, $h$ is the beam deflection. It can be deduced from Eqs. (2) and (3) that the time delay difference is

$$
\Delta t_{d}=\frac{\tau \cdot \Delta \lambda}{k} \cdot \frac{1}{h}
$$

where $\tau$ is the whole time delay of the fiber grating which is a constant determined by the grating length. The time delay difference is linearly proportional to $1 / h$. So the PAA beampointing direction is determined by the beam deflection.

\section{EXPERIMENTAL RESULTS AND DISCUSSIONS}

In the experiments, a multiwavelength laser source consisting of five commercial tunable semiconductor lasers is used. The lasers have a power of more than $3 \mathrm{~mW}$ and a full-width at half-maximum (FWHM) bandwidth of $0.015 \mathrm{~nm}$. The wavelength spacing of the lasers is $\Delta \lambda=0.3 \mathrm{~nm}$ and the center wavelength is $\lambda_{0}=1553.0 \mathrm{~nm}$. Because the proposed TTD system only needs a laser source that generates fixed wavelengths with identical wavelength spacing, a simple and economic multiwavelength fiber laser can be employed in practical systems. The chirped grating in the system is produced using a uniform FBG fabricated in single-mode photosensitive fiber using a 244-nm frequency-doubled argon ion laser source. The grating has a length of $30 \mathrm{~mm}$, a center wavelength of $1553.0 \mathrm{~nm}$, an FWHM bandwidth of about $0.23 \mathrm{~nm}$ and a peak reflectivity of higher than $99 \%$. The simply supported beam has a length of $100 \mathrm{~mm}$ and a thickness of $4.5 \mathrm{~mm}$. Its midpoint is deflected by using a high precision actuator (Newport CMA$25 \mathrm{CCCL})$. The position resolution of the actuator is $0.05 \mu \mathrm{m}$ and the maximum tuning speed is $0.4 \mathrm{~mm} / \mathrm{s}$. The TLCFG is surface-mounted onto the beam using glue that has good mechanical properties. No obvious stress hysteresis effects are observed in the experiments. It should be noted that the setup for chirp tuning is placed on a high stable optical table. For practical applications, the effect of vibration stability on the TTD system should be considered. The time-delayed light is split into five channels by a broad-band $1 \times 5$ coupler and then filtered by a bandpass filter at each channel. Each bandpass filter consists of an optical circulator and a high reflectivity FBG $(R>20 \mathrm{~dB})$. The center reflection wavelengths of the FBGs are identical to the wavelengths of the optical carriers. In practical systems, other bandpass filters can also be adopted. We should note that the reflectivity and wavelength stability of the FBGs are important factors that affect the SNR at the outputs of the photodetectors.

The reflection spectrum of the TLCFG is measured using an optical spectrum analyzer when the grating is bent. During 


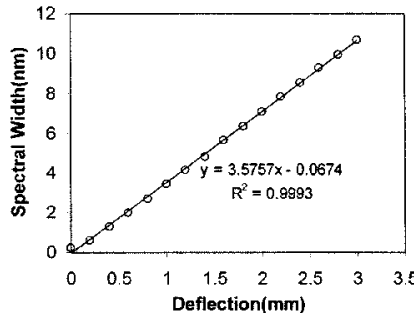

(a)

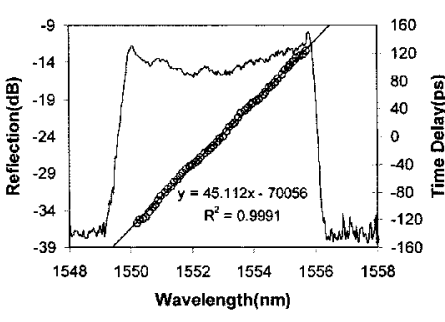

(b)
Fig. 3. Chirp tuning and time delay properties of the TLCFG (a) Spectral width as a function of beam deflection. (b) Grating reflectivity and time delay response corresponding to the beam deflection.

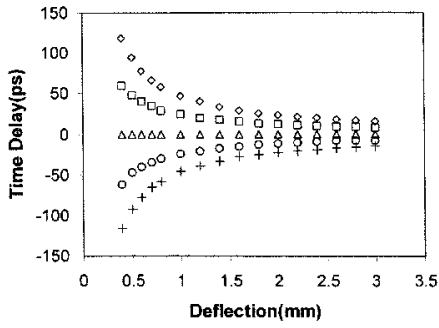

(a)

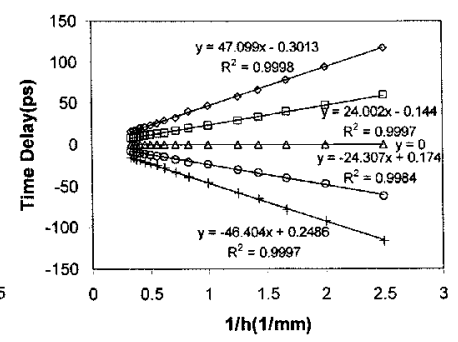

(b)
Fig. 4. Time delay responses when the TLCFG is tuned. (a) Relative time delays as a function of beam deflection. (b) Relative time delays as a function of $1 / h(+) \lambda_{1} ;(\bigcirc) \lambda_{2} ;(\Delta) \lambda_{3} ;(\square) \lambda_{4} ;(\diamond) \lambda_{5}$.

the chirp tuning, the center wavelength of the TLCFG is kept constant at the wavelength of $1553.0 \mathrm{~nm}$. Fig. 3(a) shows the spectral width of the grating as the function of beam deflection. Linear fitting of the experimental data gives a sensitivity of $3.58 \mathrm{~nm} / \mathrm{mm}$. The time delay responses are also measured experimentally when the chirp rate of the grating is tuned. As shown in Fig. 1, the light from the multiwavelength laser source is externally modulated by a $4-\mathrm{GHz} \mathrm{RF}$ signal. The modulated light enters the TLCFG from the shorter-wavelength end and reflected at different locations of the TLCFG for different wavelengths. The reflected light is again split into 5 channels, and then filtered by the bandpass filters. The signal from each bandpass filter is amplified by an EDFA and then sent to a high-speed photodetector to recover the time-delayed RF signal. The time delays are measured using an oscilloscope (Agilent $86100 \mathrm{~A})$. The time delay corresponding to $\lambda_{0}$ is selected as a reference for time delay measurements. Fig. 3(b) shows the grating reflectivity and the relative time delay response of the TLCFG when the beam deflection is $h=1.5 \mathrm{~mm}$. The ripples of the grating reflectivity may lead to the fluctuation on the signals at the outputs of the photodetectors, which can be compensated at the antenna by using electronic equalization technique [3]. The electronic equalization and amplification are widely used in PAA systems that would not add the system complexity. Fig. 4(a) shows the relative time delays selected by different wavelengths as a function of beam deflection. The curves of the time delays as a function of $1 / h$ are also shown in Fig. 4(b). Data fitting results show that the slopes of the curves are $-46.4 \mathrm{ps} \cdot \mathrm{mm},-24.3 \mathrm{ps} \cdot \mathrm{mm}$, $0,24.0 \mathrm{ps} \cdot \mathrm{mm}$ and $47.1 \mathrm{ps} \cdot \mathrm{mm}$, which are consistent with the theoretical analysis. The $R$-squared values of the data fitting are higher than 0.99 , which means a good linearity of the time delay characteristics. The small deviation of the time delay measurements away from the linearity could be attributed to the position uncertainties of the grating and the errors of time delay measurements. The experiment results also show that the SNR of the five-element TTD unit is more than $30 \mathrm{~dB}$, which meets the requirements of typical antenna end user. Thanks to the high position resolution of the actuator, the minimum time delay step can be much smaller than 1 ps. Continuous PAA beamforming can be achieved.

The available maximum number of the antenna elements is determined by the wavelength spacing of the multiwavelength laser and the spectral width of the TLCFG. The experimented five-element TTD unit can be used for PAA beamforming at microwave frequencies up to $10 \mathrm{GHz}$. At higher frequencies, the chromatic dispersion of the TLCFG will cause noticeable RF power degradation for the conventional double-sideband intensity modulation/direct detection (IM/DD) scheme (The singlesideband modulation technique should be employed for high microwave frequencies) [6], and the ripples of grating dispersion will also lead to a relative large time delay error because the needed time delay difference is much smaller at high frequencies for a given beampoint angle.

\section{CONCLUSION}

We have demonstrated a new five-element true-time-delay system that uses a single TLCFG delay line for wideband continuous TTD beamforming. The system is simple, no complicated wavelength tuning scheme is required. In addition, the synchronal control of the tunable lasers as well as the tunable bandpass filters is also avoided. Different time delays were obtained by simply tuning the TLCFG. The time delay responses were measured. The results showed that the time delays were linearly proportional to the reciprocal of the deflection, and agreed well with the theoretical analysis. The proposed system can be used for wideband continuous beamforming at radio frequencies up to $10 \mathrm{GHz}$.

\section{REFERENCES}

[1] G. A. Ball, W. H. Glenn, and W. W. Morey, "Programmable fiber-optic delay line," IEEE Photon. Technol. Lett., vol. 6, pp. 741-743, June 1994.

[2] R. A. Soref, "Fiber grating prism for true time delay beamsteering," Fiber and Integrated Optics., vol. 15, pp. 325-333, Oct. 1996.

[3] H. Zmuda, R. A. Soref, P. Payson, S. Johns, and E. N. Toughlian, "Photonics beamformer for phased array antennas using a fiber grating prism," IEEE Photon. Technol. Lett., vol. 9, pp. 241-243, Feb. 1997.

[4] A. Molony, L. Zhang, J. A. R. Williams, and I. Bennion, "Fiber Bragggrating true time-delay systems: Discrete-grating array 3-b delay lines and chirped-grating 6-b delay line," IEEE Trans. Microwave Theory Technol., vol. 45, pp. 1527-1530, Aug. 1997.

[5] J. L. Corral, J. Marti, S. Regidor, J. M. Fuster, R. Laming, and M. J. Cole, "Continuously variable true time-delay optical feeder for phased-array antenna employing chirped fiber gratings," IEEE Trans. Microwave Theory Technol., vol. 45, pp. 1531-1536, Aug. 1997.

[6] B. Ortega, J. L. Cruz, J. Capmany, M. V. Andres, and D. Pastor, "Variable delay line for phased-antenna based on a chirped fiber grating," IEEE Trans. Microwave Theory Technol., vol. 48, pp. 1352-1360, Aug. 2000.

[7] Y. Liu, J. Yang, and J. Yao, "Wideband true-time-delay unit using discrete-chirped fiber Bragg grating prism," CLEO/Pacific Rim Tech. Dig., vol. 2, pp. 28-29, July 2001.

[8] B. Ortega, J. L. Cruz, J. Capmany, M. V. Andres, and D. Pastor, "Analysis of a microwave time delay line based on a perturbation uniform fiber Bragg grating operating at constant wavelength," J. Lightwave Technol., vol. 18 , pp. 430-436, Mar. 2000.

[9] Y. Liu, J. Yao, X. Dong, and J. Yang, Tunable chirping of a fiber Bragg grating without center wavelength shift using a simply supported beam, in Opt. Eng., vol. 41, pp. 740-741, Apr. 2002. OEL 010048. 